\title{
A novel homeobox gene mediates the Dpp signal to establish functional specificity within target cells
}

\author{
Hideki Nakagoshi, ${ }^{1,2,6}$ Minako Hoshi, ${ }^{1}$ Yo-ichi Nabeshima, ${ }^{1,4}$ and Fumio Matsuzaki ${ }^{1,3,5,6}$ \\ ${ }^{1}$ Department of M olecular Genetics, N ational Institute of N euroscience, N ational Center of N eurology and Psychiatry, \\ Kodaira, Tokyo 187-8502, Japan; ${ }^{2}$ Precursory Research for Embryonic Science and Technology (PRESTO) and ${ }^{3}$ Core Research \\ for Evolutional Science and Technology (CREST) of Japan Science and Technology Corporation (JST); ${ }^{4}$ Institute for \\ Molecular and Cellular Biology, Osaka University, Suita, Osaka 565-0871, Japan; ${ }^{5}$ Department of Developmental \\ N eurobiology, Institute of Devel opment, Aging and Cancer, Tohoku U niversity, Aoba-ku, Sendai 980-8575, Japan
}

\begin{abstract}
Morphogen gradients of secreted molecules play critical roles in the establishment of the spatial pattem of gene expression. During midgut development in D rosophila, secreted molecules of Decapentaplegic (Dpp) and Wingless (Wg) establish unique transcriptional regulation within target cells to specify the resultant cell types. Here we report the identification of a novel homeobox gene, defective proventriculus (dve), which is required for the midgut specification under the control of Dpp and Wg. In dve mutants, two distinct parts of the midgut, the proventriculus and middle midgut, are abnormally organized. The Wg signal regulates dve expression during proventriculus development. On the other hand, dve is a downstream target of Dpp in the middle midgut and defines the functional specificity of copper cells along with another Dpp target gene, labial. Thus, the dve gene acts under the two distinct extracellular signals at distant parts of the midgut primordia.
\end{abstract}

[Key Words: Homeodomain; Dpp; Wg; midgut; functional specificity]

Received May 11, 1998; revised version accepted July 17, 1998.

During animal development, a mass of homogeneous cells have distinct developmental fates depending on their positions, and are organized in a stereotyped manner into a variety of functional tissues. Positional information mediated by extracellular signals plays major roles in giving rise to such a diversity of cell types within the uniform cell mass. The transforming growth factor- $\beta$ (TGF- $\beta$ ) and Wnt superfamilies (D rosophila D pp and Wg, respectively) have been well studied among secreted molecules that transmit such extracellular signals, and regulate a wide variety of cellular responses including differentiation, proliferation, adhesion, and migration (for review, see N usse and Varmus 1992; Kingsl ey 1994). Signaling by the T GF- $\beta$ superfamily is el icited by way of two types of receptor serine-threonine kinase, type-I (thick veins and saxophone in Drosophila) and type-ll (punt) (for review, see M assagué et al . 1994). Intracellular molecules essential for the signal transduction by the TGF- $\beta$ family include Smads family molecules [Mothers against dpp] (for review, see Massagué et al. 1997), and nuclear factors such as Xenopus FAST1 (Chen et al. 1996) and Drosophila schnurri (Arora et al. 1995; Grieder et al. 1995). Signaling by the Wnt superfamily is medi-

${ }^{6}$ Corresponding authors.

E-MAIL nakagosi@ncnaxp.ncnp.go.jp; fumio@ncnaxp.ncnp.go.jp; FAX (+81)-423-46-1755. ated by the Frizzled receptor family, Dishevelled, Glycogen synthase kinase-3 $\beta$ (shaggy/zeste-white 3), $\beta$ Catenin (armadillo), and nuclear factors such as TCF/ LEF-1 (pangolin) (for review, see Cadigan and Nusse 1997; Cavallo et al. 1997). Studies of Drosophila have provided valuable insights into the roles of these highly conserved signaling pathways in morphogenesis such as wing patterning (Blair 1995) and mi dgut specification (Bienz 1994; Graba et al. 1997).

The gut epithelium of Drosophila is derived from the anterior and posterior primordia at both ends of the blastoderm embryo. These primordia are initially nonsegmental and fused into a single continuum. Secreted molecules, such as D pp and Wg, induce subsequent morphogenetic events that ultimately compartmentalize the primordia into morphologically distinct sectors. During this process, these signals also act for cells to take distinct devel opmental paths to establish the functional organization of the midgut.

The proventriculus develops at the junction of the foregut and the midgut, and functions as a valve regulating the passage of food into the midgut. It is composed of three layers; the outer layer is derived from the anteriormost region of the midgut, the middle layer is derived from the foregut of mesoderm-free keyhole structure, and the inner layer is derived from the esophagus (for review, see Skaer 1993). The late steps of proventriculus 
morphogenesis are attributable to migration of cells, and are controlled by at least Hedgehog $(\mathrm{Hh})$ and $\mathrm{Wg}$, which are expressed in the mesoderm-free keyhole structure (Pankratz and Hoch 1995; Fig. 1E). Little is known so far about downstream targets that respond to $\mathrm{Hh} / \mathrm{Wg}$ signals during proventriculus development.

The midgut consists of two germ layers, the visceral mesoderm and the endoderm. The middle midgut cells derived from the endoderm differentiate into four distinct types of cells: copper, interstitial, large flat, and iron cells. These endodermal cell types are specified by $\mathrm{Dpp}$ and $\mathrm{Wg}$, which are expressed in the adhering visceral mesoderm of the parasegments (PS) 7 and 8, respectively. Copper cells exhibit a unique morphology with banana shapes and exhibit UV light-induced fluorescence after copper feeding. These characteristics are specified by a homeotic gene, labial (lab), which is activated by the $\mathrm{Dpp}$ signal in the midgut. Two different threshol ds of Wg define copper and large flat cells (Hoppler and Bienz 1995). However, it remains unclear how Lab confers the transcriptional regulation to specify copper cells.

Here, we have identified a new gene, defective proventriculus (dve), which encodes a homeodomain protein. $\mathrm{dve}^{1}$ homozygous mutants are defective in proventricu-
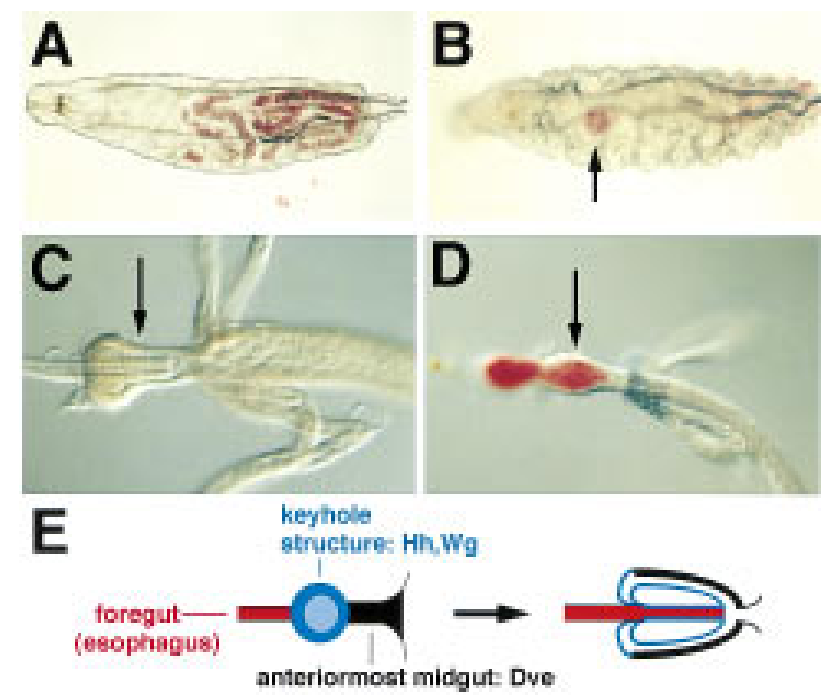

Figure 1. Proventriculus phenotypes of dve mutants. (A,B) First instar larvae of the wild type (A) or dve mutant (B) were fed for $5 \mathrm{hr}$ with a colored yeast. In dve mutants, the colored yeast is accumulated in the proventriculus (arrow in B). The magnification of $B$ is twice that of $A$. (C,D) The morphology of the proventriculus in dissected first instar larvae of the wild type (C) or dve $\mathrm{e}^{1}$ mutant (D). A rrows indicate the proventriculus. (E) Schematic representation of the proventriculus morphogenesis. The mesoderm-free keyhole structure (blue) of the foregut is most evident at stage 14. This region expresses $\mathrm{hh}$ and $\mathrm{wg}$, whose activities are essential for the subsequent migration into the anterior-most midgut (black). The anterior-most midgut expresses dve and constitutes the outer layer of the proventriculus after stage 16. The internalized foregut epithelium of the esophagus (red) and the keyhole structure constitute the inner and middle layers of the proventriculus, respectively.
Ius morphogenesis and in the arrangement of middle midgut cells. The dve gene responds differentially to the Wg or Dpp signal in the anterior-most or middl e midgut, respectively. In the anterior-most midgut, dve activity is required to maintain the three-layered structure indispensable for a functional proventriculus. In the middle midgut, the dve gene is expressed in all precursors of four distinct cell types, subsequently it is repressed only in copper cells. This repression is mediated by two Dpp target genes, lab and dve itself, and is al so essential for the functional specification of copper cells. Thus, dve is involved in different developmental aspects of the midgut under the control of the different extracellular signals. We discuss the roles of dve in the context of the network of inductive signals that organize midgut development.

\section{Results}

Identification of the dve locus

We have identified the dve locus by two enhancer-trap insertions at 58D 1-2 on the second chromosome; one is a viable allele, dve $\mathrm{SH}^{\mathrm{SH}}$, and the other is a homozygous lethal allele, dve ${ }^{1}$ (Fig. 2A). Immediately after hatching, dve ${ }^{1}$ homozygous larvae exhibit normal locomotion behavior; however, develop into small larvae and die within a day. The lethality of the dve $\mathrm{e}^{1}$ allele is attributable to the P-element insertion, because the P-element excision recovered homozygous viable adults (15 of 28). Embryos bearing dve ${ }^{1}$ in trans with $\operatorname{Df}(2 \mathrm{R}) \times 58-3$, which uncovers the dve locus, hatch normally into first instar larvae but die, suggesting that animals of this genotype have a lethal stage similar to that of $d^{1} e^{1}$ homozygotes. In addition, dve ${ }^{1}$ homozygous embryos express no detectable dve transcript (data not shown) or Dve protein (see Fig. 6B, bel ow) until stage 14; faint staining with the anti-Dve serum was detectable at early stage 17 (Fig. 3H). These observations indicate that $d^{1} e^{1}$ is a strong hypomorphic allele. We term this locus defective proventriculus based on the defects in the proventriculus formation as described below.

The reduced body sizes of dve ${ }^{1}$ homozygous larvae suggest that the feeding is affected by the dve ${ }^{1}$ mutation. The colored yeast fed to heterozygous larvae stained their guts red throughout their length (see Fig. 1A). On the other hand, it accumulated in the proventriculus in $\mathrm{dve}^{1}$ mutant larvae (arrow in Fig. 1B). Consistent with this observation, dve $\mathrm{e}^{1}$ Iarvae fail to form the proventriculus correctly (Fig. 1, cf. C and D). In the wild type, cell movement leads to formation of the internal portion of the proventriculus during embryonic stages 16-17; cells of the foregut epithelium invaginate into the anteriormost midgut that normally expresses dve (Fig. IE). In $\mathrm{dve}^{1}$ embryos, the cell migration was greatly del ayed and the internalization was only temporary (data not shown). As a result, dve ${ }^{1}$ larvae cannot form the threelayered structure of the proventriculus (Fig. 1D) as observed in hh or wg mutant embryos (Pankratz and Hoch 1995). As the dve-expressing anterior-most midgut constitutes the 
Figure 2. dve gene structure and homology al ignment. (A) Genomic organization of the dve locus. Triangles $d v e^{1}$ and $\mathrm{dve}^{\mathrm{SH} 255}$ indicate the P-element insertions. Closed boxes represent the six exons of the dve gene. (E) EcoRI; (S) Sal I. (B) Predicted amino acid sequence of the Dve protein. The region homologous to the homeodomain is indicated in yellow and the three helices are underlined. The putative transcriptional activation domain of an acidic amino acid cluster is indicated in red. (C) Homology alignment with known homeodomain proteins. Identical amino acids are indicated in yellow. Dashes indicate gaps introduced to maximize similarity. The positions of $\alpha$ helices are indicated below: (\#) Invariant amino acids; (*) highly conserved amino acids in homeodomains. The ninth amino acid of the recognition helix (helix 3 ) is indicated by a red arrowhead. The amino acids ( $Q, Y$, and $R$ ) marked by green are highly conserved in homeodomains except for POU homeodomains. Invariant amino acids in POU homeodo-

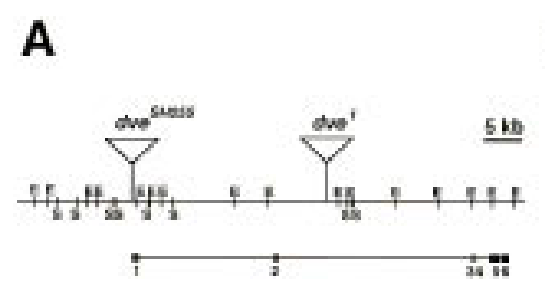

B

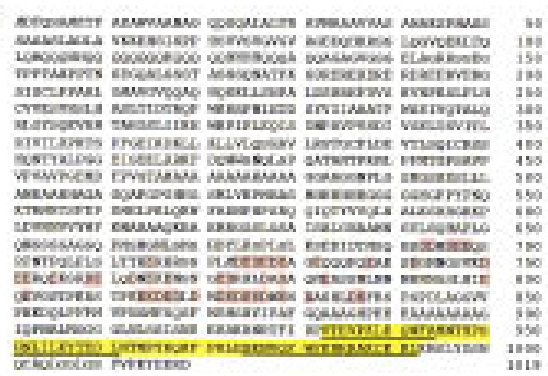

C

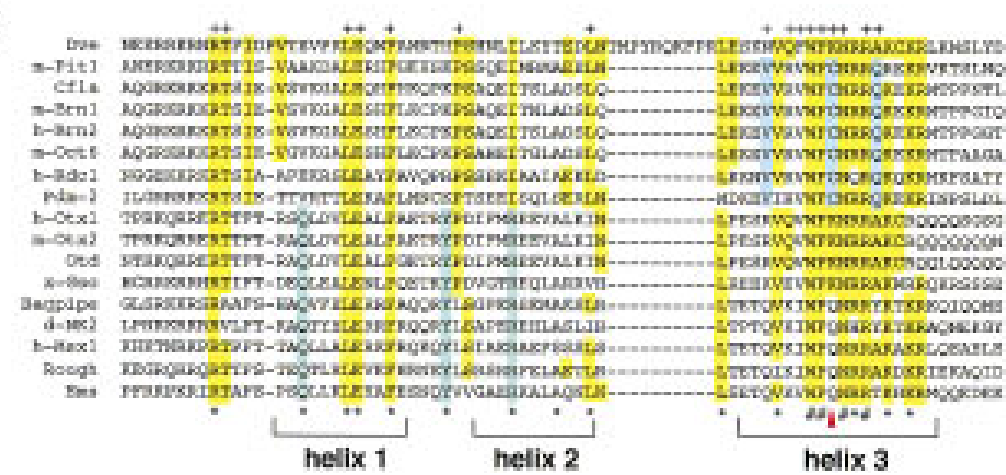

mains (Herr and Cleary 1995) are indicated above by plus signs ( + ), and the blue amino acids (,$C$, and Q) are conserved in POU homeodomains but not in other types of homeodomains. The homeodomain sequences are from the following sources: $\mathrm{m}$-Pit1 (Bodner et al. 1988; Ingraham et al. 1988), Cfla (Johnson and Hirsh 1990), m-Brn1 (Hara et al. 1992), h-Brn2 (Oct3N; He et al. 1989; Schreiber et al. 1993), m-Oct6 (M eijer et al. 1990; Suzuki et al. 1990), h-Rdc1 (Collum et al. 1992), Pdm-2 (Billin et al. 1991; Lloyd and Sakonju 1991), h-Otx1, m-Otx2 (Simeone et al. 1993), Otd (Finkelstein et al. 1990), x-Gsc (Cho et al. 1991), Bagpipe (d-N K3), d-N K2 (Kim and Nirenberg 1989), h-M sx1 (Hox-7; Hewitt et al. 1991), Rough (Kimmel et al. 1990), and Ems (Dalton et al. 1989).

outer layer of the proventriculus (Fig. 1E), these dve ${ }^{1}$ phenotypes suggest that dve activity is required for the functional development of outer layer cells to retain the internal portion of the proventriculus.

\section{Molecular cloning of the dve gene}

To isolate the dve gene, we used genomic DNAs flanking the dve $\mathrm{SH}^{\mathrm{SH} 25}$ insertion and identified the full-length cDNA of a $4.9-\mathrm{kb}$ transcript. Analysis of the genomic DNA of the dve gene reveal ed that the transcript consists of six exons and that the P-element of dve $\mathrm{eH}^{\mathrm{SH}} \mathrm{C5}$ is inserted at $167 \mathrm{bp}$ upstream from the transcription start site. The P-element insertion of the lethal $\mathrm{dve}^{1}$ allele is located within the second intron of the dve gene (Fig. 2A).

The predicted open reading frame of Dve is 1019 amino acids long. Homology with the homeodomain is found near its carboxy-terminal region (yellow in Fig. 2B). The Dve homeodomain contains all four invariant amino acids located within helix 3 (sharps in Fig. 2C), and matches well with other highly conserved residues (asterisks in Fig. 2C; Gehring 1992). However, the Dve homeodomain is unusual, as it has a 10-amino-acid insertion between helices 2 and 3 (Bürgl in 1997). Theninth amino acid of helix 3 confers the recognition specificity for its binding sequences (red arrowhead in Fig. 2C; Hanes and Brent 1989; Treisman et al. 1989), and the recognition helix of Dve is closest to the orthodenticle (otd) class homeodomain. In contrast, helices 1 and 2 of Dve exhibit homology with POU homeodomains rather than otd class homeodomains (Fig. 2C). Therefore, the Dve homeodomain seems to be a novel class of homeodomain that is intermediate between POU and otd class homeodomains. The Dve staining observed in the nucleus is consistent with its homology with the homeodomain and suggests that Dve is a putative transcription factor.

\section{Expression pattern of dve in the embryonic midgut}

The expression of the Dve protein exhibits a pleiotropic pattern including three separate domains of the midgut: anterior-most, middle, and posterior-most regions. Because defects in the lethal dve ${ }^{1}$ allele are evidently observed in the gut, we focus on dve expression during gut development. At stage 10, dve expression is first observed in the invaginated stomodeum (Fig. 3A), where hh and wg are expressed under the control of the wingedhelix transcription factor Fork head (Hoch and Pankratz 1996). We compared anti-Dve staining with wg gene expression that was monitored with wg-lacZ. The expression of dve and wg overlaps initially in the stomodeum, and is then segregated into nonoverlapping but adjacent regions; the mesoderm-free keyhole structure expresses $\mathrm{wg}$, whereas dve is solely expessed in the anterior-most region of the midgut (Fig. 4A,E), which becomes outer layer of the proventriculus later at stage 16 (Fig. $4 \mathrm{H}$ ). 


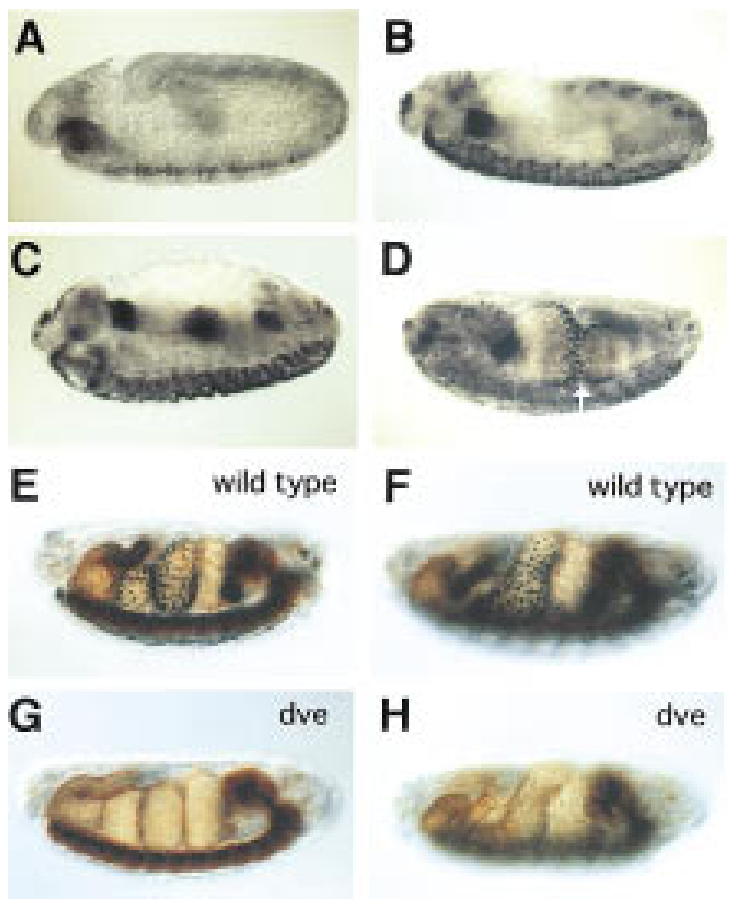

Figure 3. Dve expression in the embryonic midgut. Lateral view of wild-type $(A-F)$ or dve ${ }^{1}$ mutant embryos $(G, H)$ stained with anti-Dve serum (A-D; anterior is left and dorsal is up). The morphology of the gut was visualized by double staining with BP102 (E-H; brown signal). In E-H, the right side of embryos is visualized (anterior is right) so as to better show the overall morphology of the gut. (A-C) Wild-type embryos at stages 11 , 12 , and 13, respectively. (D) A stage-15 embryo. The arrow indicates the position of the middle constriction. (E,G) Stage-16 embryos of the wild type $(E)$ and $d v e^{1}$ mutant $(G)$, respectively. $(\mathrm{F}, \mathrm{H})$ Stage-17 embryos of the wild type $(\mathrm{F})$ and dve $\mathrm{e}^{1}$ mutant $(\mathrm{H})$, respectively.
From stage 13 onward, dve is also expressed in two other parts of the endodermal midgut; one is the middle regi on where the anterior and posterior midgut primordia are fused, and the other is the posterior-most region (see Fig. 3C). The middle region expressing dve corresponds to both the anterior and posterior sides of the middle constriction (arrow in Fig. 3D). At stage 16, this region becomes the second and third gut lobes (Fig. 3E). Dve-expressing cells in this region include copper cell precursors that coexpress lab (see Fig. 7A,D, bel ow).

\section{wg signal regulates dve expression during proventriculus development}

As the dve-expressing domain in the presumptive proventriculus initially overlaps with the wg- and hh-expressing domain (Fig. 4A), we examined the possibility that $\mathrm{Wg}$ or $\mathrm{Hh}$ regulates the expression of the dve gene. In wg $^{\mathrm{CX} 4}$ null embryos, dve expression is abolished in the stomodeum, whereas epidermal expression is normally detected (Fig. 4B). In hhAC mutant embryos, dve is normally expressed (Fig. 4C). Thus, Wg rather than $\mathrm{Hh}$ is required to activate the dve gene in the presumptive proventriculus.

The keyhole structure al so expresses cubitus interruptus (ci). In $\mathrm{ci}^{\mathrm{D}}$ mutants, the expression of $\mathrm{wg}$ and $\mathrm{hh}$ is not maintained, leading to the absence of a keyhole structure. The maintenance of $\mathrm{wg}$ expression also requires hh activity (Pankratz and Hoch 1995). In ci ${ }^{\mathrm{D}}$ mutants, dve expression is never detected in the presumptive proventriculus until stage 13 (Fig. 4D), whereas it begins to be detectable at stage 14 despite the absence of a keyhole structure (Fig. 4F). In addition, dve expression expands ectopically into the presumptive first gut lobe after stage 15 (Fig. 4G). Similar ectopic expression of dve
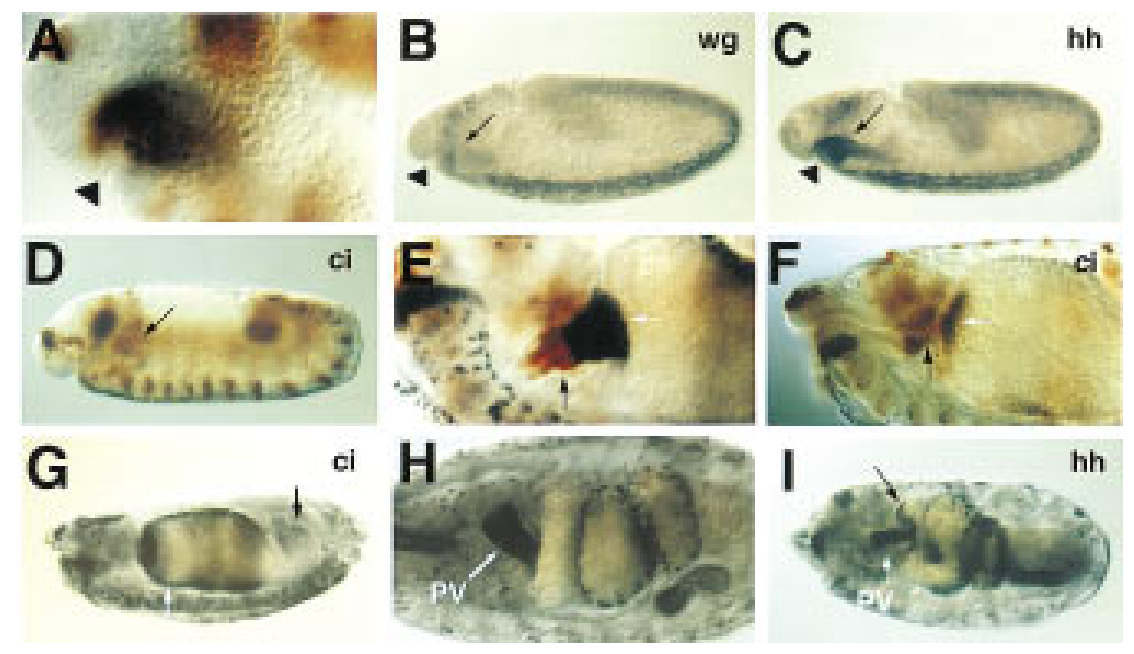

Figure 4. Wg signal regulates dve expression in the proventriculus. Embryos were stained with anti-Dve serum (black) and anti- $\beta$-gal (brown in A,D-F). (A-C) Dve expression in the invaginated stomodeum overlapped with wg-lacZ expression (brown in A) at stage 11 , and was abolished in $\mathrm{w}^{\mathrm{CX} 4}$, but not in hh $\mathrm{h}^{\mathrm{AC}}$ mutants (arrows in B,C, respectively). Arrowheads indicate the stomodeum invagination. (D) In cubitus interruptus $\left(\mathrm{ci}^{\mathrm{D}}\right)$ mutants, the expression of $\mathrm{wg}$ and hh is not maintained and dve expression is al so undetectable at stage 12 (arrow). (E)The mesoderm-free keyhole structure of the wild type (stage 14 ) expresses wg-lacZ (black arrow), and the adjacent region expresses dve (white arrow). (F) In $\mathrm{ci}^{\mathrm{D}}$ mutants, the keyhole structure is not formed (black arrow); however, dve expression begins to be detectable in the anterior-most region of the stage-14 midgut (white arrow). $(\mathrm{G})$ In $\mathrm{ci}^{\mathrm{D}}$ mutants at stage 15 , dve expression expands posteriorly (white arrow) in contrast to the great reduction in the posterior-most region of the midgut (black arrow). $(\mathrm{H}, \mathrm{I}) \mathrm{Dve}$ expression in the proventriculus (PV) of a wild-type (stage 16) and a hh $\mathrm{h}^{\mathrm{J} 35}$ mutant embryo (stage 17) is indicated (white arrow in $\mathrm{H}$ and $\mathrm{I}$, respectively). In hh' $\mathrm{h}^{1 / 35}$ mutants, dve expression appears to expand posteriorly (black arrow). $(A-G)$ Lateral view; $(H, I)$ ventral view. Anterior is left in all panels. 
is observed in hh' ${ }^{1 / 35}$ or wg ${ }^{1 \mathrm{~L} 114}$ mutant embryos (Fig. 4l; data not shown). The ectopic dve expression in these mutants suggests that the $\mathrm{Wg}$ signal is also required to define the posterior border of late dve expression in the presumptive proventriculus, in addition to the initial activation of the dve gene.

\section{Dpp signal regulates dve expression} in the middle midgut

In the middle midgut, $\mathrm{Dpp}$ and $\mathrm{Wg}$ signal s from the visceral mesoderm control gene expression on both the anterior and posterior sides of the middle constriction. Hence we examined whether dve expressi on depends on the D pp or Wg signals in this region. In thick veins (tkv) mutants that lack the functional type I receptor for Dpp, dve expression is completely absent in the middle midgut, whereas the expression in other parts of the midgut is not affected (Fig. 5, cf. A with C). This indicates that dve expression in the middle midgut requires the $D p p$ signal. On the other hand, the ubiquitous expression of $\mathrm{dpp}$ throughout the visceral mesoderm, using the combination of UAS-dpp and 24B-Gal 4 driver, induced dve expression throughout the underlying endoderm (Fig. 5, cf. A with E). This dependence of dve expression on Dpp is quite similar to that of lab expression (StaehlingHampton and Hoffmann 1994).

We used abdominal-A (abd-A) mutants to examine the dependence of dve expression on $\mathrm{Wg}$. In abd-A mutants, the expression of U Itrabithorax (U bx) and dpp in the visceral mesoderm expands throughout the posterior midgut and endogenous wg is not activated (Immergl ück et al. 1990; Panganiban et al. 1990; Reuter et al. 1990). In abd-A mutants, dve expression also expands posteriorly (Fig. 5D). These three observations are consistent with the notion that Dpp is sufficient to induce dve expression without $\mathrm{Wg}$ in the midgut. To confirm this, we examined the effect of the ubiquitous wg expression in the visceral mesoderm on dve expression. The ubiquitous activation of the $\mathrm{Wg}$ signal using shaggy null mutations induces the expression of $U b x$ and dpp only anteriorly, but not posteriorly, in the midgut ( $\mathrm{Yu}$ et al. 1996). The ubiquitous wg expression in the whole visceral mesoderm induced ectopic dve expression only anteriorly (Fig. 5F), indicating that this induction appears to be indirect and mediated by ectopic dpp activation. It is noteworthy that the ectopic dve induction is especially strong in the anterior-most region that is normally responsi ve to $\mathrm{Wg}$ (Fig. 5F). Taken together, these results indicate that dve expression in the middle midgut does not depend on Wg but on Dpp, which is in contrast to dve expression during proventriculus development.

Two distinct transcription factors, schnurri (shn) and Mad, have been reported to mediate the Dpp signal (Arora et al. 1995; Grieder et al. 1995; Kim et al. 1997). The expression of Dve protein in the middle midgut is absent in shn mutants (Fig. 5G; data not shown). In addition, the ubiquitous mesodermal expression of dpp in the shn mutant background fails to induce endodermal dve expression (Fig. 5G), indicating that dve induction
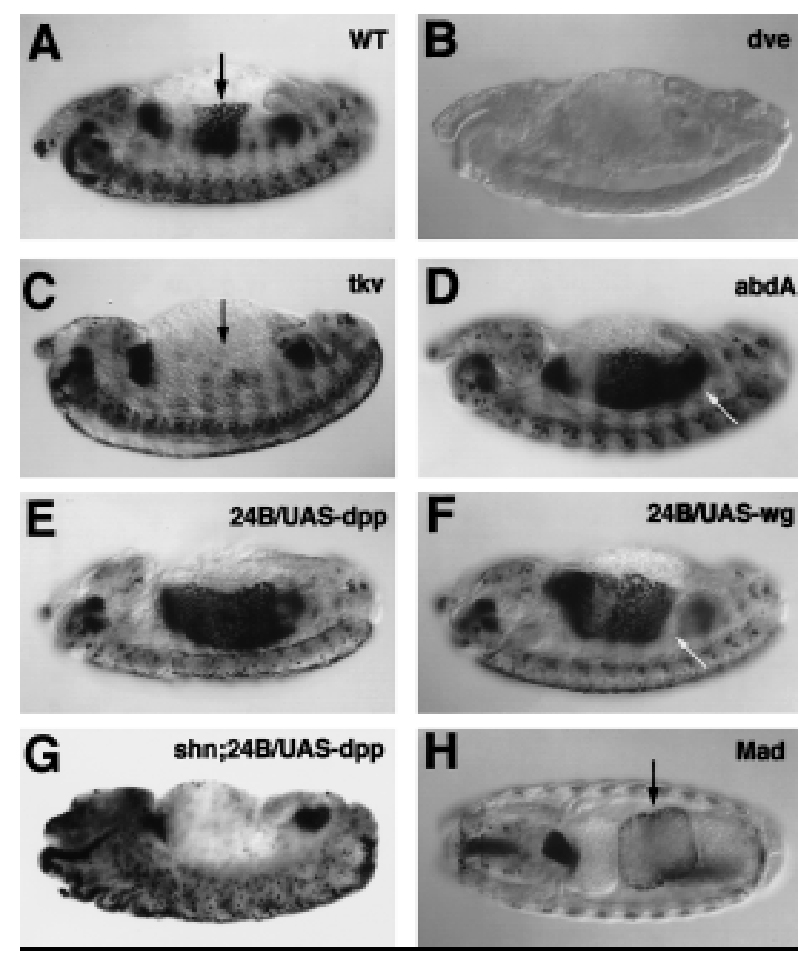

Figure 5. Dpp signal regulates dve expression in the middle midgut. Lateral $(A-G)$ or ventral $(H)$ view of embryos stained with anti-Dve serum. Anterior is left in all panels. (A) A wildtype embryo (stage 14). The arrow indicates the middle midgut expression. (B) dve ${ }^{1}$ homozygous embryos at stage 14 are not stained at all. (C) Dve expression in the middle midgut (arrow) is absent in thick veins $\left(\mathrm{tkv}^{8}\right)$ mutant embryos. (D) Dve expression is expanded throughout the posterior midgut (arrow) in abdominal-A (abd-A ${ }^{\mathrm{M}}$ ) mutants. $(E, F)$ Ectopic expression of $\mathrm{dpp}$ and wg was induced in the whole visceral mesoderm on the crossing of UAS-dpp $(E)$ and UAS-wg $(F)$, respectively, with 24B-Gal4. N ote the absence of dve induction in the posterior midgut (arrow in F), and the strong posterior expansion in the anterior-most region. (G) In schnurri $\left(\mathrm{shn}^{1}\right)$ mutants, dpp was induced ubiquitously in all the visceral mesoderm, whereas dve expression was absent in the middle midgut. $(H)$ In Mothers against dpp ( $\mathrm{Mad}^{12}$ ) mutants, dve is normally expressed in the middl e midgut, al though the middle constriction does not occur at stage 16 (indicated by an arrow).

requires shn activity in the midgut endoderm. In contrast, dve expression is not affected in Mad mutants, whereas lab expression is compl etely absent in the same mutant background (Fig. 5H; see N ewfeld et al. 1996 and also Discussion).

Phenotypes of dve mutants in the larval middle midgut

Although the dve gene is strongly expressed in the embryonic middle midgut, morphological defects are not evident in this region in dve ${ }^{1}$ mutants; gut constriction normally occurs, and the arrangement of the stage-17 midgut appears to be normal (see Fig. 3E-H). Cells that express the dve gene at embryonic stages develop into four types of larval midgut cells: copper, interstitial, 


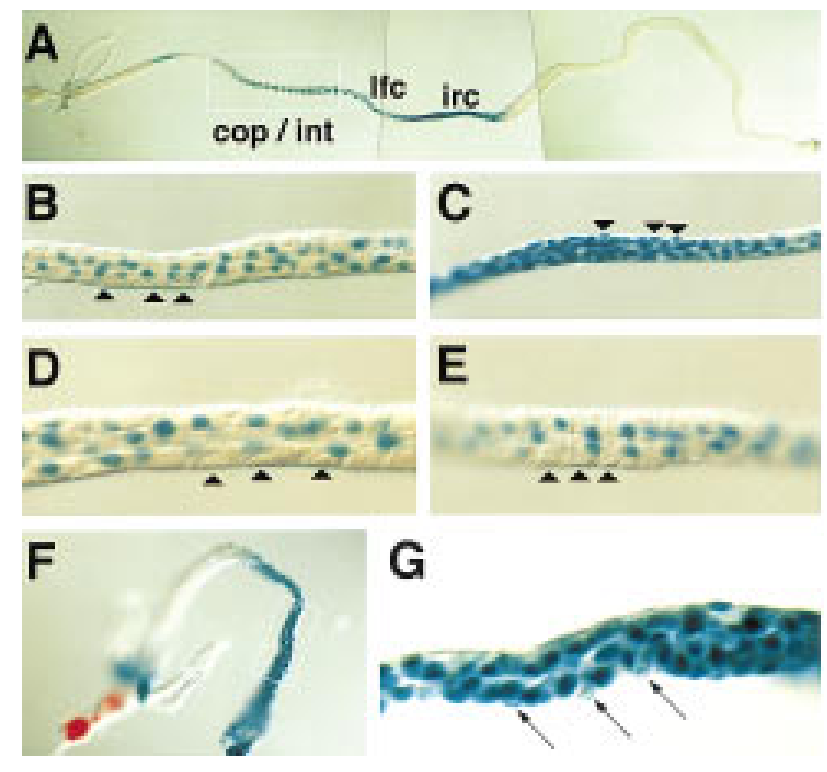

Figure 6. Phenotypes of dve mutants in the larval midgut. The expression of lacZ from the $\mathrm{dve}^{1}$ allele (dve ${ }^{1}-\mathrm{lacZ}$ ) or an interstitial cell marker (C5-2-7) was examined by $X$-gal staining. (A) Interstitial, large flat (Ifc), and iron cells (irc) are stained in the midgut prepared from heterozygous $\mathrm{dve}^{1 / C y O}$ larvae. The copper and interstitial cell region (cop/int) is boxed. (B,C) High magnification views of copper and interstitial cell regions from dve $/$ CyO (B) and dve $/$ Df(2R)X58-3 mutant larvae $(C)$, respectively. Arrowheads indicate the positions of copper cells. (D,E) The expression of an interstitial cell marker, C5-2-7, was examined in C5-2-7, dve ${ }^{\mathrm{E} 3} / \mathrm{CyO}$ (D) and C5-2-7, dve ${ }^{\mathrm{E3}} / \mathrm{Df}(2 \mathrm{R}) \times 58-3$ mutant larvae $(E)$, respectively. Arrowheads indicate the positions of copper cells. $(F, G)$ Rescue experiments on a mutant phenotype of ectopic dve $\mathrm{e}^{1}-\mathrm{lac} Z$ in copper cells. The exogenous dve transgene was induced at embryonic stage 17 under the control of heat shock promoter-Gal 4 (hs-Gal4) in the dve $\mathrm{Cu}^{\mathrm{m}} \mathrm{-}$ tant background. (F) The abnormal morphology of the proventriculus in a dve ${ }^{1}$ homozygous mutant was visualized by colored yeast feeding. (G) High magnification view of the boxed region in $\mathrm{F}$ shows that the ectopic $\mathrm{dve}^{1}$-lacZ expression is partially suppressed in copper cells (arrows). Anterior is left in all panels.

large flat, and iron cells. The expression of the dve gene in these larval cells was monitored as the expression of the lac $Z$ gene that is located in the dve ${ }^{1}$ enhancer-trap insertion (dve $\mathrm{e}^{1}-\mathrm{lacZ}$ ). $\beta-$ Galactosidase activity attributable to dve ${ }^{1}-\mathrm{lac} Z$ is observed in interstitial, large flat, and iron cells, but not in copper cells in heterozygous larvae (Fig. 6A,B). In dve mutant larvae, we found the ectopi $\mathrm{c}$ dve $\mathrm{e}^{1}-\mathrm{lac} Z$ expression in copper cells, in addition to highly disorganized arrangement of these cells (Fig. $6 C$ ). These observations suggest that the dve activity is required to repress its own expression in copper cells. To determine whether the dve gene could rescue this mutant phenotype of ectopic dve $\mathrm{e}^{1}-\mathrm{lac} Z$ expression, the dve gene was induced ubiquitously in the dve ${ }^{1}$ mutant background under the control of Gal4-UAS system (Brand and Perrimon 1993). Weak dve induction in stage-17 embryos suppressed the ectopic dve ${ }^{1}-$ lacZ expression in larval copper cells (Fig. 6C, F,G), indicating that a mutant phenotype is exactly caused by the lack of the dve gene product.

To monitor cell types in the larval midgut, the C5-2-7 enhancer-trap insertion, which marks specifically interstitial cells, was introduced into the dve ${ }^{\mathrm{E} 38}$ background (see $M$ aterials and $M$ ethods). In dve ${ }^{E 38}$ mutants, C5-2-7 expression remained unaffected although the arrangement of copper cells was highly disorganized (Fig. 6D,E), suggesting that interstitial cells are not strongly affected by the dve mutation.

In the larval midgut, the dve gene is thus expressed in a cell type-specific manner that requires its own activity. As described below, the repression of the dve gene in copper cells depends on the function of both Lab and Dve itself, and is essential for the functional specification of these cells.

Copper cells are specified by the cross-regulation between dve and lab

In the middle midgut, lab is expressed under the control of $\mathrm{Dpp}$ as is dve, whereas lab is regulated negatively by the Wg signal to generate a sharp posterior border (Hoppler and Bienz 1995). We compared the expression of lab and dve using lab upstream 6.3 kb-lacZ (lab-6.3lacZ), which mimics endogenous lab expression (Tremml and Bienz 1992). The expression of lab is observed in the endoderm just beneath the dpp-expressing visceral mesoderm of PS 7, but not in the inner endodermal cells as described (Reuter et al. 1990; Fig. 7B). In contrast, dve is expressed more broadly throughout the inner endodermal layers including presumptive interstitial cell precursors (Fig. 7A-C). A nother difference in lab and dve expression is that dve expression is repressed subsequently in lab-expressing cells that become copper cells (see Fig. $6 \mathrm{~B})$. We examined the possibility that Lab might be involved in the repression of dve in copper cells. In lab mutants, dve ${ }^{\mathrm{E} 8}$-lacZ expression is not repressed in presumptive copper cells as observed in dve mutants (Fig. 7E-G). This pattern of gene expression is similar to that of neighboring interstitial cells, which express dve continuously without lab expression in the wild type. Hence the inability of dve repression in the presumptive copper cells of lab mutants could be attributable to a change in cell fate-copper cells to interstitial cells. In this case, the expression of an interstitial cell marker C5-2-7 should expand in this region. However, it turned out that its expression is abolished in lab mutants (Fig. $7 \mathrm{H}, \mathrm{I})$, suggesting that interstitial cells are also affected by the lab mutation. Thus, it is unlikely that the lab mutation causes the transformation of copper cells into interstitial cells. Taken together, the repression of dve requires the activities of both Lab and Dve itself.

To determine whether the dve repression in copper cells is essential for establishment of their correct identity, dve was overexpressed ubiquitously at stage 17. Strong heat shock-induced dve expression resulted in an abnormal morphology of copper cells. The typical banana shape of copper cells is frequently lost, the cells be coming circular (Fig. 7J), suggesting an abnormal cyto- 


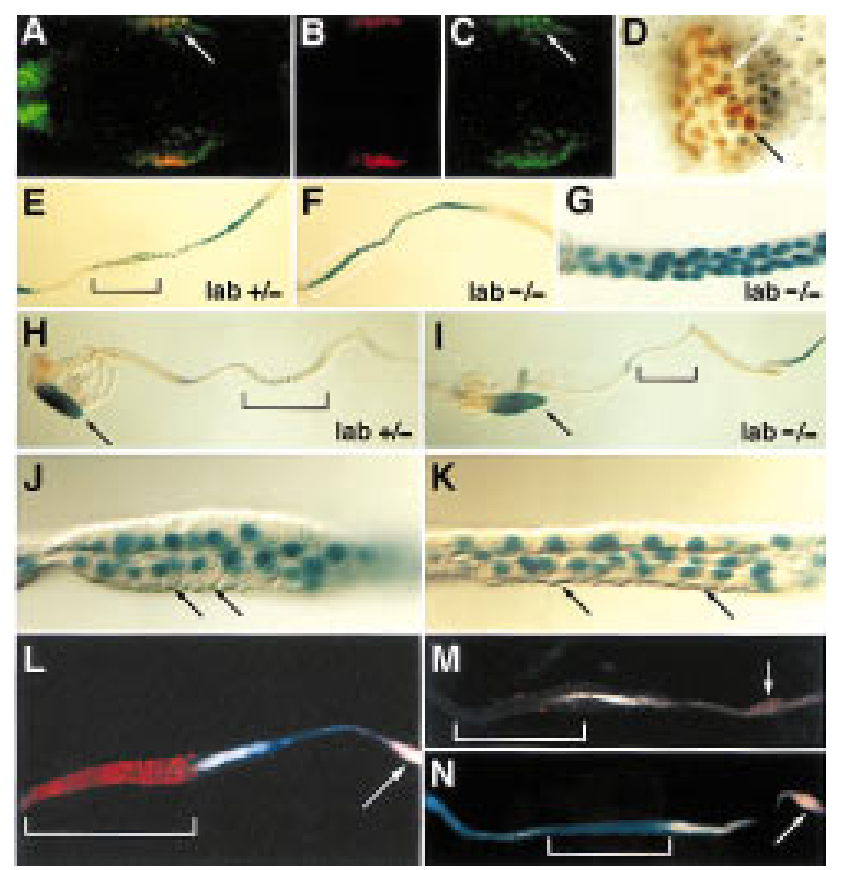

Figure 7. Functional specification of copper cells by Dve and Lab. (A-C) Confocal images of lab-6.3lacZ (red in B) and dve (green in C) expression at stage 14 (dorsal view). A merged image is shown in A. Arrows indicate a Dve-positive cell located in the inner layer of the endoderm. (D) Coexpression of dve (black) and lab-6.3lacZ (brown) in copper cell precursors (black arrow) at stage 16 (ventral view). Interstitial cell precursors (Dve singlepositive; white arrow) and copper cell precursors are intermingled. (E-G) The expression of dve ${ }^{\mathrm{E} 8}-\mathrm{lacZ}$ in lab ${ }^{\mathrm{vd1}}$ heterozygous larvae $(E)$ and homozygous mutants $(F, G)$, respectively. The copper and interstitial cell regions are boxed. (G) High magnification view of the boxed region in $\mathrm{F} .(\mathrm{H}, \mathrm{I})$ The lac $\mathrm{Z}$ expression of interstitial cell marker C5-2-7 in lab ${ }^{\text {vd1 }}$ heterozygous larvae $(\mathrm{H})$ and homozygous mutants $(\mathrm{I})$, respectively. The lab mutation abolishes C5-2-7 expression in the midgut (boxed region); however, it does not affect the expression in the ventral nerve cord (arrows). (J,K) X-gal staining of guts from dve $1 /+$; hs-GAL4/UAS-dve-9B2 larvae heat shocked at embryonic stage $17\left(\mathrm{~J}, 37^{\circ} \mathrm{C}\right.$ for $60 \mathrm{~min}$; K, $37^{\circ} \mathrm{C}$ for $30 \mathrm{~min}$ ). (L-N) UVinduced fluorescence after copper feeding. Guts from dve $1 /+$; hs-GAL4 /+ (L) and dve $/$ +; hs-GAL4/UAS-dve-9B2 larvae $(\mathrm{M}, \mathrm{N})$ heat shocked at embryonic stage $17\left(37^{\circ} \mathrm{C}\right.$ for $\left.30 \mathrm{~min}\right)$ were excited with UV light. The copper and interstitial cell regions are boxed. Control guts exhibit strong fluorescence of copper cells (L). Ectopic dve expression greatly reduces the fluorescence of copper cells $(\mathrm{M}, \mathrm{N})$. $\mathrm{N}$ ote the presence of posterior fluorescence corresponding to the iron cell regions (arrows). Anterior is left in all panels.

skeletal organization in these cells. To determine the effect of ectopic dve on the copper cell function, ubiquitous dve expression was induced alternatively by a milder heat shock. Under this condition, the copper cells appear to retain their normal morphology (Fig. 7K), however, the typical character of copper cells, UV light-induced orange fluorescence on copper feeding, is greatly reduced in these guts (Fig. 7L-N). This mild heat shock does not affect the posterior fluorescence attributable to iron cells, suggesting that the function of copper cells is specifically impaired by this treatment. Taken together with the results for dve mutants described above, both the loss of function and ectopic expression of the dve gene affect the morphology of copper cells, and ectopic dve expression impairs the function of copper cells without affecting their morphol ogy. Unfortunately, the function of copper cells in dve mutants could not be examined because of the feeding defect in the proventriculus; however, the morphological abnormality observed in dve mutants suggests that dve activity is also required for the functional devel opment of copper cell precursors. Our results indicate that temporally restricted dve re pression is essential for this functional specification in addition to the lab gene, which is indispensable for copper cell development (Hoppler and Bienz 1994). This repression depends on Lab and Dve itself. Thus, the crossregulation of the two $D$ pp target genes lab and dve specifies the functional identity of copper cells.

\section{Discussion}

Dve expression is regulated differentially by $\mathrm{Dpp}$ and $\mathrm{Wg}$

Accumulating evidence has shown that signal inputs from $\mathrm{Dpp}$ and $\mathrm{Wg}$ exert cooperative or antagonistic effects to define the border of target gene expression. The development of the leg imagi nal disc has provided a typical example for the antagonistic and cooperative functions of these two factors. dpp and wg are expressed adjacently on the anterior side of the anterior-posterior compartment boundary of the disc; wg is expressed ventrally, whereas dpp is expressed at high levels dorsally. The convergence of both signals is required to induce Distal-less and aristaless at the center of the disc (Campbell et al. 1993; Cohen et al. 1993). On the other hand, the expression of a ventral marker, $\mathrm{H} 15$, is activated by $\mathrm{Wg}$ and repressed by $\mathrm{Dpp}$. Thus, the antagonistic effects of these two factors restrict the $\mathrm{H} 15$ expression ventrally (Brook and Cohen 1996). Another example of the complex gene regulation by $\mathrm{Dpp}$ and $\mathrm{Wg}$ has been found in midgut morphogenesis, where both dpp and wg are expressed in adjacent regions of the visceral mesoderm of PS 7 and 8, respectively. Dpp and Wg act independently but synergistically on the visceral mesoderm enhancer of U bx; however, Wg-dependent transcriptional stimulation requires the Dpp signal (Riese et al. 1997). In contrast, the lab expression in the endoderm is activated by Dpp and repressed by Wg to confer the discrete boundary between copper and large flat cells (Hoppler and Bienz 1995).

The roles of Dpp and Wg in dve expression are quite different from those reported previously. Dve-expressing domains are exposed to both $\mathrm{Wg}$ and $\mathrm{Dpp}$; however, dve expression responds differentially to either of them in distinct parts of the midgut. In the middle midgut, dve expression depends mainly on $\mathrm{Dpp}$, although a possible contribution of the Wg signal cannot be excluded, as observed for U bx expression. In contrast, the dve expression in the anterior-most midgut depends on $\mathrm{Wg}$, but not 
on Dpp. In this region, the posterior border of dve expression appears to be defined by the Wg signal.

How does the dve gene sort the $\mathrm{Wg}$ and $\mathrm{Dpp}$ signals depending on the domains of the midgut? Enhancer elements responding to the two signals in the midgut have been well studied for the $U$ bx gene. In the case of the $U$ bx gene, the two signal inputs are mediated by the $B$ enhancer, which includes the Dpp response sequence (DRS) and the Wg response sequence (WRS). These two response sequences are closely located so that signal inputs from Dpp and Wg coordinately induce the expression in a pattern spanning both sides of the middle constriction in the visceral mesoderm (Riese et al. 1997). This expression pattern is similar to that of the dve gene, although the $B$ enhancer of the $U$ bx gene drives the expression mainly in the visceral mesoderm. Interestingly, four copies of this enhancer derivative (L-CRE) drive Wgdependent expression in the anterior-most endoderm of the midgut where the dve gene is activated. The similarity in the spatial pattern between dve expression and the $U$ bx enhancer activation raises the possibility that $\mathrm{Dpp}$ and $\mathrm{Wg}$ act on similar regulatory elements that are present in the dve gene to induce the broad expression in the middle midgut. We do not know anything about the regulatory mechanisms that activate dve gene depending on Wg but not on Dpp in the anterior-most midgut. Characterization of the regulatory el ements of the dve gene would provide useful clues for spatial regulation during Dpp- and Wg-dependent pattern formation.

Early gradient of Dpp defines the cell identity in the midgut

In the middle midgut, both the lab and dve genes are activated by the Dpp signal, but they do not respond equally to D pp. Iab expression is restricted strictly to the endodermal cells that are in contact with the dpp-expressing visceral mesoderm (Reuter et al. 1990). In contrast, dve is widely activated even in endodermal cells that are remote from the D pp source (Fig. 7A-C). Assuming a concentration gradient of $\mathrm{Dpp}$ in the endoderm as well as wing disc (Lecuit et al. 1996; Nellen et al. 1996), these observations suggest that lab expression requi res a higher concentration of Dpp than dve expression. Therefore, dve can be activated by low levels of Dpp that are insufficient to induce lab. Furthermore, we found a difference between lab and dve gene activation in the requirement for Mad, which transduces the Dpp signal. Mutant embryos for Mad fail to express lab but show normal activation of dve ( $\mathrm{N}$ ewfeld et al. 1996; Fig. 5H). If the maternal supply of Mad transcripts is sufficient to induce dve, but not lab expression, the difference in $\mathrm{M}$ ad dependence might be explained by the different thresholds for the Dpp signal between Iab and dve activation.

The early gradient of Dpp in the endoderm thus gives rise to two distinct cells in terms of gene expression; one is $\mathrm{lab}^{-}$and dve ${ }^{+}$, and the other is $\mathrm{lab}^{+}$and $\mathrm{dve}^{+}$(Fig. 8). If this situation is disrupted by ubiquitous lab induction during 7-9 hr of embryonic development, interstitial cells are transformed into copper cell-like cells that ex-

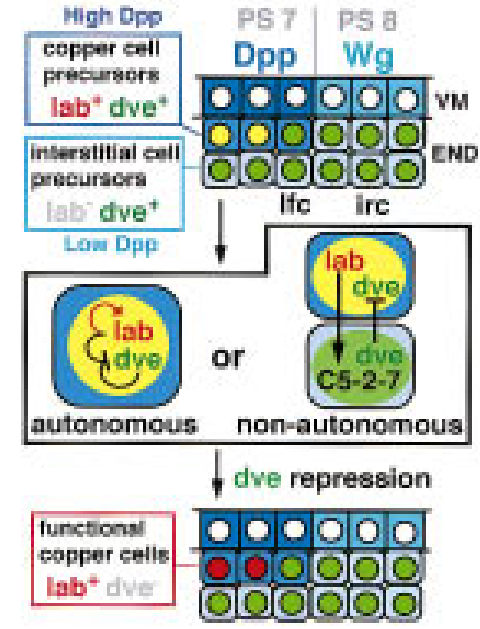

Figure 8. Model for the functional specification of copper cells by Dve and Lab. Schematic representation of gene expression during midgut development. High levels of D pp can induce the expression of lab (red) and dve (green) in the endoderm (END) just beneath the PS 7 visceral mesoderm (VM). These cells indicated by yellow nucleus become to copper cells (copper cell precursors; $\left.\mathrm{lab}^{+}, \mathrm{dve}^{+}\right)$. M ore inner and posterior endodermal cells, which are exposed to low levels of Dpp, can express dve but not lab (green nucleus; lab-, dve+), and become to interstitial, large flat (Ifc) and iron cells (irc). Copper cell precursors coexpress dve and lab, and subsequently repress dve expression (red nucleus; $\mathrm{Iab}^{+}$, dve-) in a Lab- and Dve-dependent manner. Two alternative mechanisms of dve repression (cell autonomous and cell nonautonomous) are boxed in the middle. After stage 17 , the Lab function is cell nonautonomously required for the expression of interstitial cell marker C5-2-7. The dve repression mediated by Lab and Dve itself is critical for acquisition of the functional specificity in larval copper cells (see text for details).

hibit orange fluorescence on copper feeding (Hoppler and Bienz 1994). At later stages, these two types of cells intermingle, and interstitial cell precursors are also exposed to high Dpp (Fig. 7D; Reuter et al. 1990). Nonetheless, these cells never express lab at later stages, indicating that the cell identity once established by the early D pp gradient seems to be maintained. M echanisms involved in this maintenance of cell identity remain unclear. One of the simplest explanations would be that Dve continues to suppress lab in interstitial cell precursors. However, this is unlikely because ectopic expression of lab-6.3lacZ is not detectable in dve mutants (data not shown). A nother possibility is that the negative autoregulation of lab is involved in the continuous lab suppression in interstitial cell precursors. In fact, heat shock induction of the lab transgene between 9 and $11 \mathrm{hr}$ of development frequently results in severe repression of copper cell development, suggesting that heat-induced Lab during a critical period suppresses the endogenous lab gene (Hoppler and Bienz 1994). The time window for this negative lab autoregulation coincides well with when interstitial cell precursors are exposed to high D pp. It is also an intriguing possibility that Dpp-induced Smad molecules, which inhibit the TGF- $\beta$ /Dpp signal, 
partici pate in these processes as a negative feedback loop (Hayashi et al. 1997; N akao et al . 1997; T suneizumi et al. 1997).

\section{Dve function in copper cell specification}

Dve is expressed in all precursors of four distinct types of middle midgut cells, and is repressed subsequently only in copper cells. This repression is mediated by Lab and Dveitself, and turns out to be essential for the functional specification of copper cells, as judged by the ability of copper uptake. This does not necessarily mean that dve activity is required for the cell fate decision of copper cells. In fact, the expression of several cell-type markers for middl e midgut cells is unaffected in dve mutants (Fig. $6 \mathrm{E}$; data not shown). dve activity seems to be required to refine the functional specificity at later stages of development after the cell fate decision has been completed.

It is very important to understand the mechanism of dve repression that is critical for the acquisition of functional identity in copper cells. There are two possibilities that explain how the repression of the dve gene occurs in larval copper cells (Fig. 8). One is a cell nonautonomous mechanism; interstitial cells might send a signal to adjacent copper cells to repress dve gene expression. For instance, an interstitial cell marker, C5-2-7, is abolished in lab mutants (Fig. $7 \mathrm{H}, \mathrm{I}$ ), suggesting a cell nonautonomous interaction between interstitial cells and copper cells. The other is a cell autonomous mechanism by which the dve gene is autoregul ated negativel y in copper cells. In copper cell precursors, Lab and Dve are coexpressed during embryogenesis, and are able to act coordinately as transcriptional regulators. Thus, the latter possibility is more plausible, and Dve might function as a transcriptional cofactor for Lab to specify the functional identity of copper cells. Further characterization of the dve gene would clarify the molecular mechanism conferring the functional specificity to each of the four distinct types of middle midgut cells.

\section{Materials and methods}

Fly strains

The following mutant strains were used: $\mathrm{Df}(2 \mathrm{R}) \times 58-3$ (Kerre brock et al. 1995) uncover the dve locus; $\mathrm{wg}^{\mathrm{CX} 4}, \mathrm{wg}^{\mathrm{IL114}}, \mathrm{hh}^{\mathrm{AC}}$, $\mathrm{hh}^{\mathrm{IJ} 35}, \mathrm{ci}^{\mathrm{D}}, \mathrm{tkv}^{8}, \mathrm{abd}^{\mathrm{M}} \mathrm{A}^{\mathrm{1}}, \mathrm{shn}^{1}, \mathrm{Mad}^{12}$, and lab ${ }^{\mathrm{vd1}}$ (FLYBASE; http://morgan.harvard.edu/). dpp or wg was induced ectopically in the embryonic mesoderm by using 24B-Gal4, UAS-dpp (Staehling-Hampton and Hoffmann 1994) and UAS-wg (Lawrence et al. 1995). To monitor the cell type-specific expression in the midgut, copper cell marker lab-6.3lacZ (Tremml and Bienz 1992), interstitial cell marker C5-2-7, and large flat cell markers A3-2-66 and B1-2-49 (Hartenstein and Jan 1992; Hoppler and Bienz 1995) were used. The following lacZ balancers were used to distinguish homozygous embryos: en ${ }^{11} \mathrm{CyO}, \mathrm{Cy}$ Oftz-lacZ, TM 3ftz-lacZ, and TM 3 U bx-lacZ. The $\mathrm{ci}^{\mathrm{D}}$ homozygous embryos were identified as to the expression pattern of wg-lacZ derived from en ${ }^{11} \mathrm{CyO}$. dve $\mathrm{SH}^{\mathrm{SH} 5}$ and $\mathrm{dve}^{1}$ are enhancer-trap lines of $F Z$ and $P Z$, respectively; dve ${ }^{1}$ is identical to I(2)0173801738 generated by the Berkeley Drosophila Genome Project (Karpen and Spradling 1992; M lodzik and Hiromi 1992). By the P-element excision of $d^{2} e^{1}$, an imprecise excision allele $\mathrm{dve}^{\mathrm{E} 38}$ was recovered that has the internal deletion of both the rosy marker and the lacZ gene. dve ${ }^{\mathrm{E} 38}$ mutants showed essentially the same Dve protein expression and phenotype as the $d^{1} e^{1}$ allele. To examine roles in cell-type specification, each of the midgut cell type markers was introduced into the dve $\mathrm{E}^{\mathrm{E} 38}$ background by recombination. dve $\mathrm{E}^{\mathrm{E} 8}$ is a hypomorphic allele that lacks the rosy marker but retains the lac $Z$ gene, and this allele was used to monitor dve expression in the lab mutant background.

A 4.5-kb EcoRI fragment of dve cDN A was inserted into the pUAST vector (Brand and Perrimon 1993) to construct pUASdve, and several independent transformants were obtained. Of these lines, UAS-dve-9B2 (homozygous viable on the third chromosome) was used in this study. For the rescue experiments, yw; dve / CyO [y ${ }^{+}$; hs-GAL4 flies were crossed with y w; $\mathrm{dve}^{1} / \mathrm{CyO}\left[\mathrm{y}^{+}\right.$]; U AS-dve-9B2, and stage-17 embryos were heat shocked at $35^{\circ} \mathrm{C}$ for $30 \mathrm{~min}$. Homozygous first instar larvae were identified from the absence of the yellow $(y)$ marker. For ectopic expression, yw; dve $/ \mathrm{CyO}^{\mathrm{C}} \mathrm{y}^{+}$]; U AS-dve-9B2 flies were crossed with hs-Gal4, and yw; dve ${ }^{1} / \mathrm{CyO}^{2} \mathrm{y}^{+}$; hs-GAL4 flies were crossed with Oregon- $R$ as a control, and stage-17 embryos were heat shocked at $37^{\circ} \mathrm{C}$. Subsequently, the gut morphology and copper fluorescence were examined.

\section{Isolation of genomic and CDNA clones}

The dve gene was identified originally from an enhancer trap line that shows an abnormal object fixation response (dve $\mathrm{e}^{\mathrm{SH} 255}$; H. Nakagoshi and F. Matsuzaki, unpubl.). A 4.5-kb Sall genomic fragment flanked to dve $\mathrm{SH}^{\mathrm{SH} 255}$ insertion was used for screening Oregon-R adult head cD N A library. A nearly full-length dve cDNA clone (W12) was isolated, and its $5^{\prime}$ end was determined by 5 ' RACE. Genomic clones covering the dve locus were isolated from Oregon-R genomic DN A library, and the exon-intron boundaries were determined. DN A sequencing was carried out using an ABI 373 DNA sequencer (Applied Biosystems).

\section{Antibody staining}

A 2.3-kb EcoRI fragment of dve cDNA was inserted into the pTrcHisC vector (Invitrogen Xpress System). As a result, the carboxy-terminal 368 amino acid fragment was fused to the amino-terminal histidine tag. The resultant fusion protein (HE26) was purified and immunized to a rabbit. The HE26 antiserum was used for further immunostaining experiments. The stages of embryonic development are according to Campos-Ortega and Hartenstein (1985). For whole-mount staining, embryos were dechorionized and then fixed in a $4 \%$ formal dehyde/ phosphate-buffered saline (PBS): heptane $=1: 1$ solution for 30 min, devitellinized with methanol and then washed with PBS$0.2 \%$ Tween 20 . Embryos were incubated with HE26 antiserum (1:1000 dilution) in the blocking solution ( $5 \%$ skim milk/PBS$0.2 \%$ Tween 20), and then washed with PBS-0.2\% Tween 20. For the detection, a secondary antibody (biotin-conjugated antirabbit IgG, diluted 1:1000; Vector Labs) and a horseradish peroxidase (HRP)-conjugated avidin-biotin complex solution (ABC Elite; Vector Labs) were used. To detect lacZ expression, an anti- $\beta$-gal actosidase monoclonal antibody (diluted 1:1000; Promega) was used.

For confocal microscopic observations with a Bio-Rad MRC1024, Cy-3 conjugated anti-mouse IgG and Cy-5 conjugated anti-rabbit IgG (Jackson) were used as secondary antibodies.

Feeding assay and X-gal staining of larval guts

For the feeding assay, larvae were allowed to grow on sucrosesupplied agar plates of $100-\mathrm{mm}$ dish with a red-colored yeast (dyed with carmine; Sigma). Several hours feeding was enough 
to visualize gut motility. To analyze the copper cell function, larvae were fed with the yeast containing a high concentration $(2-3 \mathrm{mg} / \mathrm{ml})$ of $\mathrm{CuSO}_{4}$ for at least $5 \mathrm{hr}$ to visualize posterior fluorescence attributable to iron cells. The guts of dissected larvae were immediately mounted in $80 \%$ glycerol and excited with UV light using a DAPI filter. The procedure was basically according to Hoppler and Bienz (1994).

For X-gal staining, first instar larvae were dissected in a fixing solution (1\% glutaral dehyde/PBS), and subsequently their guts were washed with PBS and stained in $0.1 \%$ 5-bromo-4-chloro3 -indolyl- $\beta$-galactopyranoside (X-gal) for $\beta$-galactosidase activity. The stained guts were washed with PBS-0.2\% Tween 20 and then mounted in $80 \%$ glycerol.

\section{Acknowledgments}

We are grateful to M. Bienz for lab-6.3lacZ strain and comments on the manuscript; T.L. Orr-Weaver, S. Cohen, C. N üsslein-Volhard, A. Bejsovec, S. Goto, S. Hayashi, T. Tabata, T. Uemura, Y. Takamatsu, and the stock centers of Bloomington, Bowling Green and U mea for fly strains; R. M urakami for helpful discussion. We are al so grateful to A. Fujisawa-Sehara for the initial indication of the embryonic expression of the dve gene. We also thank $T$. Jinnai and $H$. Izumi for their technical assistance, C. Hama for the genomic DN A library, and all members of our laboratory for helpful discussion and encouragement. This work was partly supported by grants to $\mathrm{H}$. N. from PRESTO (Precursory Research for Embryonic Science and Technology) of Japan Science and Technology Corporation.

The publication costs of this article were defrayed in part by payment of page charges. This article must therefore be hereby marked 'advertisement' in accordance with 18 USC section 1734 solely to indicate this fact.

\section{Note}

The nucl eotide sequence data reported in this paper will appear in the DDBJ, EMBL, and GenBank nucleotide sequence databases under the following accession number AB010299.

\section{References}

Arora, K., H. Dai, S.G. Kazuko, J. Jamal, M.B. O'Connor, A. Letsou, and R. Warrior. 1995. The Drosophila schnurri gene acts in the Dpp/TGF $\beta$ signal ing pathway and encodes a transcription factor homologous to the human MBP family. Cell 81: 781-790.

Bienz, M. 1994. Homeotic genes and positional signaling in the Drosophila viscera. Trends Genet. 10: 22-26.

Billin, A.N., K.A. Cockerill, and S.J. Poole. 1991. Isolation of a family of Drosophila POU domain genes expressed in early development. Mech. Dev. 34: 75-84.

Blair, S.S. 1995. Compartments and appendage development in Drosophila. BioEssays 17: 299-309.

Bodner, M., J.-L. Castrillo, L.E. Theill, T. Deerinck, M. Ellisman, and M. Karin. 1988. The pituitary-specific transcription factor GHF-1 is a homeobox- containing protein. Cell 55: 505-518.

Brand, A.H. and N. Perrimon. 1993. Targeted gene expression as a means of altering cell fates and generating dominant phe notypes. Development 118: 401-415.

Brook, W.J. and S.M. Cohen. 1996. Antagonistic interactions between wingless and decapentaplegic responsible for dorsal-ventral pattern in the Drosophila leg. Science 273: 13731377.

Bürglin, T.R. 1997. Analysis of TALE superclass homeobox genes (MEIS, PBC, KNOX, Iroquois, TGIF) reveals a novel domain conserved between plants and animals. Nucleic Acids Res. 25: 4173-4180.

Cadigan, K.M. and R. N usse. 1997. Wnt signaling: A common theme in animal development. Genes \& Dev. 11: 32863305.

Campbell, G., T. Weaver, and A. Tomlinson. 1993. Axis specification in the devel oping Drosophila appendage: The role of wingless, decapentaplegic, and the homeobox gene aristaless. Cell 74: 1113-1123.

Campos-Ortega, J.A. and V. Hartenstein. 1985. The embryonic development of Drosophila melanogaster. Springer-Verlag, Berlin, Germany.

Cavallo, R., D. Rubenstein, and M. Peifer. 1997. Armadillo and dTCF: A marriage made in the nucleus. Curr. O pin. Genet. Dev. 7: 459-466.

Chen, X., M.J. Rubock, and M. Whitman. 1996. A transcriptional partner for MAD proteins in TGF- $\beta$ signalling. Nature 383: 691-696.

Cho, K.W., B. Blumberg, H. Steinbeisser, and E.M. De Robertis. 1991. M olecular nature of Spemann's organizer: The role of the Xenopus homeobox gene goosecoid. Cell 67: 1111-1120.

Cohen, B., A.A. Simcox, and S.M. Cohen. 1993. Allocation of the thoracic imaginal primordia in the Drosophila embryo. Development 117: 597-608.

Collum, R.G., P.E. Fisher, M. Datta, S. Mellis, C. Thiele, K. Huebner, C.M. Croce, M.A. Israel, T. Theil, T. Moroy, R. DePinho, and F.W. Alt. 1992. A novel POU homeodomain gene specifically expressed in cells of the developing mammalian nervous system. Nucleic Acids Res. 20: 4919-4925.

Dalton, D., R. Chadwick, and W. McGinnis. 1989. Expression and embryonic function of empty spiracles: A Drosophila homeo box gene with two patterning functions on the anterior-posterior axis of the embryo. Genes \& Dev. 3: 1940-1956.

Finkelstein, R., D. Smouse, T.M. Capaci, A.C. Spradling, and N. Perrimon. 1990. The orthodenticle gene encodes a novel homeo domain protein involved in the devel opment of the Drosophila nervous system and ocellar visual structures. Genes \& Dev. 4: 1516-1527.

Gehring, W.J. 1992. The homeobox in perspective. Trends Biochem. Sci. 17: 277-280.

Graba, Y., D. Aragnol, and J. Pradel. 1997. Drosophila Hox compl ex downstream targets and the function of homeotic genes. BioEssays 19: 379-388.

Grieder, N.C., D. N ellen, R. Burke, K. Basler, and M. Affolter. 1995. Schnurri is required for Drosophila Dpp signaling and encodes a zinc finger protein similar to the mammalian transcription factor PRDII-BF1. Cell 81: 791-800.

Hanes, S.D. and R. Brent. 1989. DN A specificity of the bicoid activator protein is determined by homeodomain recognition helix residue 9. Cell 57: 1275-1283.

Hara, Y., A.C. Rovescalli, Y. Kim, and M. Nirenberg. 1992. Structure and evolution of four POU domain genes expressed in mouse brain. Proc. Natl. Acad. Sci. 89: 3280-3284.

Hartenstein, V. and Y.N. Jan. 1992. Studying Drosophila embryogenesis with P-lacZ enhancer trap lines. Wilhelm Roux's Arch. Dev. Biol. 201: 194-220.

Hayashi, H., S. Abdollah, Y. Qiu, J. Cai, Y.-Y. Xu, B.W. Grinnell, M.A. Richardson, J.N. Topper, M.A. Gimbrone Jr., J.L. Wrana, and D. Falb. 1997. The MAD-related protein Smad7 associates with the TGF $\beta$ receptor and functions as an antagonist of TGF $\beta$ signaling. Cell 89: 1165-1173.

He, X., M.N. Treacy, D.M. Simmons, H.A. Ingraham, L.W. Swanson, and M.G. Rosenfeld. 1989. Expression of a large family of POU-domain regulatory genes in mammal ian brain development. Nature 340: 35-41. 
Herr, W. and M.A. Cleary. 1995. The POU domain: Versatility in transcriptional regulation by a flexible two-in-one DN Abinding domain. Genes \& Dev. 9: 1679-1693.

Hewitt, J.E., L.N. Clark, A. Ivens, and R. Williamson. 1991. Structure and sequence of the human homeobox gene HOX7. Genomics 11: 670-678.

Hoch, M. and M.J. Pankratz. 1996. Control of gut development by fork head and cell signaling molecules in Drosophila. Mech. Dev. 58: 3-14.

Hoppler, S. and M. Bienz. 1994. Specification of a single cell type by a Drosophila homeotic gene. Cell 76: 689-702.

- - - 1995. Two different thresholds of wingless signalling with distinct developmental consequences in the Drosophila midgut. EMBO J. 14: 5016-5026.

Immerglück, K., P.A. Lawrence, and M. Bienz. 1990. Induction across germ layers in Drosophila mediated by a genetic cascade. Cell 62: 261-268.

Ingraham, H.A., R. Chen, H.J. Mangalam, H.P. Elsholtz, S.E. Flynn, C.R. Lin, D.M. Simmons, L. Swanson, and M.G. Rosenfeld. 1988. A tissue-specific transcription factor containing a homeodomain specifies a pituitary phenotype. Cell 55: 519-529.

Johnson, W.A. and J. Hirsh. 1990. Binding of a Drosophila POUdomain protein to a sequence element regulating gene expression in specific dopaminergic neurons. N ature 343: 467470.

Karpen, G.H. and A.C. Spradling. 1992. Analysis of subtelomeric heterochromatin in the Drosophila minichromosome Dp1187 by single P element insertional mutagenesis. Genetics 132: 737-753.

Kerrebrock, A.W., D.P. Moore, J.S. Wu, and T.L. Orr-Weaver. 1995. Mei-S332, a Drosophila protein required for sisterchromatid cohesion, can localize to meiotic centromere regions. Cell 83: 247-256.

Kim, J., K. Johnson, H.J. Chen, S. Carroll, and A. Laughon. 1997. Drosophila Mad binds to DNA and directly mediates activation of vestigial by Decapentapl egic. Nature 388: 304-308.

Kim, Y. and M. Nirenberg. 1989. Drosophila NK-homeobox genes. Proc. Natl. Acad. Sci. 86: 7716-7720.

Kimmel, B.E., U. Heberlein, and G.M. Rubin. 1990. The homeodomain protein rough is expressed in a subset of cells in the devel oping Drosophila eye where it can specify photoreceptor cell subtype. Genes \& Dev. 4: 712-727.

Kingsley, D.M. 1994. The TGF- $\beta$ superfamily: N ew members, new receptors, and new genetic tests of function in different organisms. Genes Dev. 8: 133-146.

Lawrence, P.A., R. Bodmer, and J.-P. Vincent. 1995. Segmental patterning of heart precursors in Drosophila. Development 121: 4303-4308.

Lecuit, T., W.J. Brook, M. Ng, M. Calleja, H. Sun, and S.M. Cohen. 1996. Two distinct mechanisms for long-range patterning by Decapentaplegic in the Drosophila wing. Nature 381: 387-393.

Lloyd, A. and S. Sakonju. 1991. Characterization of two Drosophila POU domain genes, related to oct-1 and oct- 2 , and the regulation of their expression patterns. Mech. Dev. 36: 87-102.

Massagué, J., L. Attisano, and J.L. Wrana. 1994. The TGF- $\beta$ family and its composite receptors. Trends Cell Biol . 4: 172-178.

Massagué, J., A. Hata, and F. Liu. 1997. TGF- $\beta$ signaling through the Smad pathway. Trends Cell Biol. 7: 187-192.

Meijer, D., A. Graus, R. Kraay, A. Langeveld, M.P. Mulder, and G. Grosveld. 1990. The octamer binding factor Oct6: cDNA cloning and expression in early embryonic cells. Nucleic Acids Res. 18: 7357-7365.

Mlodzik, M. and Y. Hiromi. 1992. Enhancer trap method in
Drosophila: Its application to neurobiology. Methods Neurosci. 9: 397-414.

N akao, A., M. Afrakhte, A. M orén, T. N akayama, J.L. Christian, R. Heuchel, S. Itoh, M. Kawabata, N.E. Heldin, C.H. Heldin, and P. ten Dijke. 1997. Identification of Smad7, a TGF $\beta$-inducible antagonist of TGF- $\beta$ signalling. Nature 389: 631-635.

N ellen, D., R. Burke, G. Struhl, and K. Basler. 1996. Direct and long-range action of a DPP morphogen gradient. Cell 85: 357-368.

N ewfeld, S.J., E.H. Chartoff, J.M. Graff, D.A. M elton, and W.M. Gelbart. 1996. Mothers against dpp encodes a conserved cytoplasmic protein required in DPP/TGF- $\beta$ responsive cells. Devel opment 122: 2099-2108.

N usse, R. and H.E. Varmus. 1992. Wnt genes. Cell 69: 10731087.

Panganiban, G.E., R. Reuter, M.P. Scott, and F.M. Hoffmann. 1990. A Drosophila growth factor homolog, decapentaplegic, regulates homeotic gene expression within and across germ layers during midgut morphogenesis. Development 110: 1041-1050.

Pankratz, M.J. and M. Hoch. 1995. Control of epithelial morphogenesis by cell signaling and integrin molecules in the Drosophila foregut. Devel opment 121: 1885-1898.

Reuter, R., G.E. Panganiban, F.M. Hoffmann, and M.P. Scott. 1990. Homeotic genes regulate the spatial expression of putative growth factors in the visceral mesoderm of Drosophila embryos. Development 110: 1031-1040.

Riese, J., X. Yu, A. Munnerlyn, S. Eresh, S.-C. Hsu, R. Grosschedl, and M. Bienz. 1997. LEF-1, a nucl ear factor coordinating signal ing inputs from wingl ess and decapentaplegic. Cell 88: 777-787.

Schreiber, E., A. Tobler, U. Mali piero, W. Schaffner, and A. Fontana. 1993. cDN A cloning of human N-Oct3, a nervous-system specific POU domain transcription factor binding to the octamer DNA motif. Nucleic Acids Res. 21: 253-258.

Simeone, A., D. Acampora, A. M allamaci, A. Stornaiuolo, M.R. D'A pice, V. N igro, and E. Boncinelli. 1993. A vertebrate gene related to orthodenticle contains a homeodomain of the bicoid class and demarcates anterior neuroectoderm in the gastrulating mouse embryo. EMBO J. 12: 2735-2747.

Skaer, H. 1993. The alimentary canal. In The development of Drosophila melanogaster (ed. M. Bate, and A. Martinez A rias), pp. 941-1012. Cold Spring Harbor Laboratory Press, Cold Spring Harbor, NY.

Staehling-Hampton, K. and F.M. Hoffmann. 1994. Ectopic decapentaplegic in the Drosophila midgut al ters the expression of five homeotic genes, dpp, and wingless, causing specific morphological defects. Dev. Biol. 164: 502-512.

Suzuki, N., H. Rohdewohld, T. Neuman, P. Gruss, and H.R. Schöler. 1990. Oct-6: A POU transcription factor expressed in embryonal stem cells and in the developing brain. EMBO J. 9: 3723-3732.

Treisman, J., P. Gönczy, M. Vashishtha, E. Harris, and C. Desplan. 1989. A single amino acid can determine the DNA binding specificity of homeodomain proteins. Cell 59: 553562.

Tremml, G. and M. Bienz. 1992. Induction of Iabial expression in the Drosophila endoderm: Response elements for dpp signalling and for autoregulation. Development 116: 447-456.

Tsuneizumi, K., T. Nakayama, Y. Kamoshida, T.B. Kornberg, J.L. Christian, and T. Tabata. 1997. Daughters against dpp modulates dpp organizing activity in Drosophila wing development. Nature 389: 627-631.

Yu, X., S. Hoppler, S. Eresh, and M. Bienz. 1996. decapentaplegic, a target gene of the wingless signalling pathway in the Drosophila midgut. Development 122: 849-858. 


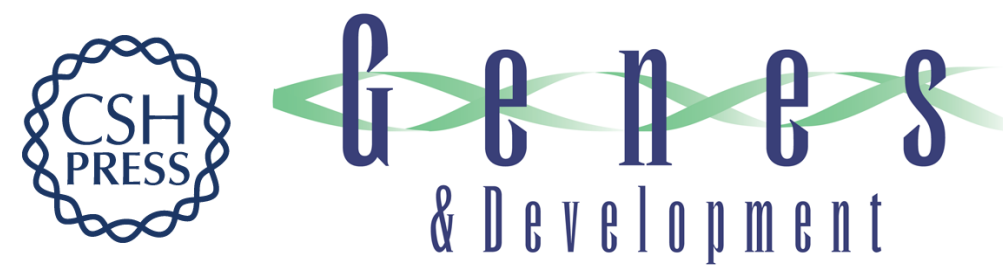

\section{A novel homeobox gene mediates the Dpp signal to establish functional specificity within target cells}

Hideki Nakagoshi, Minako Hoshi, Yo-ichi Nabeshima, et al.

Genes Dev. 1998, 12:

Access the most recent version at doi:10.1101/gad.12.17.2724

References This article cites 61 articles, 19 of which can be accessed free at: http://genesdev.cshlp.org/content/12/17/2724.full.html\#ref-list-1

License

Email Alerting

Receive free email alerts when new articles cite this article - sign up in the box at the top Service right corner of the article or click here.

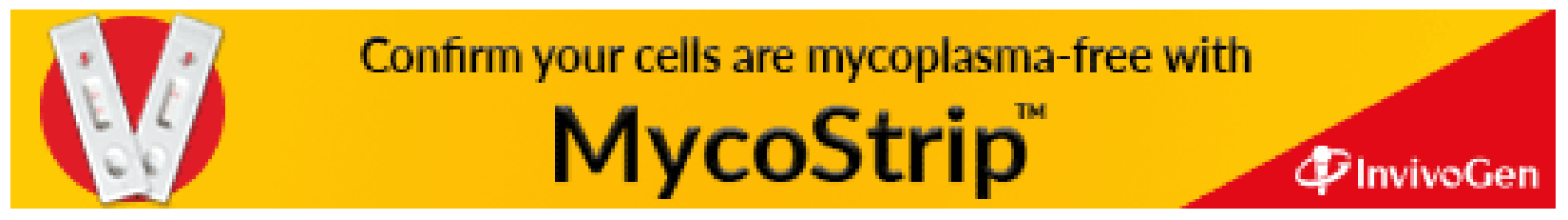

